\title{
Repeat lung cancer screenings reveal less CXR abnormalities with different variations than first-time screening
}

Tatsuo Kimura $^{1 *}$, Shinya Fukumoto ${ }^{1}$, Akemi Nakano ${ }^{1}$, Hideki Fujii ${ }^{1}$, Yuji Nadatani ${ }^{1}$, Yukie Tauchi ${ }^{1}$, Shingo Takashima ${ }^{1}$, Yuko Nishii ${ }^{1}$, Koji, Otani $^{2}$, Naomi Ageshio ${ }^{3}$, Tomohiro Suzumura ${ }^{4}$ and Norifumi Kawada ${ }^{1,5}$

${ }^{1}$ Department of Premier Preventive Medicine, Graduate School of Medicine, Osaka City University, Osaka, Japan

${ }^{2}$ Department of Gastroenterology, Graduate School of Medicine, Osaka City University, Osaka, Japan

${ }^{3}$ Department of Diagnostic and Interventional Radiology, Graduate School of Medicine, Osaka City University, Osaka, Japan

${ }^{4}$ Department of Clinical Oncology, Graduate School of Medicine, Osaka City University, Osaka, Japan

${ }^{5}$ Department of Hepatology, Graduate School of Medicine, Osaka City University, Osaka, Japan

\begin{abstract}
Objectives: Chest radiography (CXR) is the most common screening procedure for the detection of lung cancer. A comparative CXR review can help to detect new shadows. Participants (pts) who undergo repeated screening at the same facility as the health check-up can receive a comparative review of the CXR. It has been hypothesized that the diagnosis of CXR abnormalities in screening would be different when compared to previous CXR and when not.

Methods: We retrospectively analyzed one-year cohort derived from the ongoing MedCity21 health check-up registry. The pts with abnormalities requiring medication in CXR were invited to our clinic for chest computed tomography (CT). We compared the varieties of abnormalities on CXR between repeated and first-visit pts using chi-square tests.

Results: A total of 13,690 repeated / first visits of 7872 / 5818 (57.5 / 42.5\%) pts were enrolled. The CXR abnormalities included 324 of 143 / 191 pts and 234 of 108 / 126 pts who underwent chest CT, respectively. Repeated pts had a significantly lower proportion of abnormalities in CXR that required medication than the first-visit pts ( $<$ 0.01). CT confirmation revealed that CXR abnormalities in repeated pts were diagnosed with different variations compared to those of first-visit pts. Repeated pts had a significantly lower proportion of old inflammatory changes $(\mathrm{p}=0.02)$ and a higher proportion of acute inflammatory shadow ( $\mathrm{p}=0.02)$ than first-visit pts.
\end{abstract}

Conclusions: Immediate confirmation by CT revealed that CXR abnormalities in repeated pts were diagnosed with different variations compared to those of firsttime pts. A comparative review of previous CXR may be highly effective in ruling out lung cancer.

Abbreviations: CXR: Chest X ray; pts: participants, CT: computed tomography; LDCT: low dose CT, GGO: Ground glass opacity; MLP: Mayo Lung Project

\section{Introduction}

Lung cancer is the third most common form of cancer and the leading cause of cancer-related deaths in both men and women in Japan. Smoking, exposure to secondhand smoke, a family history of lung cancer, procedures leading to exposure to radiation such as imaging tests, environmental exposure to air pollution, and workplace exposure to asbestos and arsenic can all increase the risk of lung cancer [1]. Although avoiding risk factors may lower patient risk, it does not mean that all lung cancers can be prevented. As people age, they spend more time exposed to environmental factors, and their chances of developing cancer increase accordingly. Therefore, routine health check-ups are important. Early diagnosis improves cancer outcomes by providing care at the earliest possible stage.

The American Cancer Society recommends yearly lung cancer screening with an low-dose CT (LDCT) for certain people at higher risk of lung cancer who meet the following conditions: aged 55 to 74 years and in good health, currently smoking or having quit smoking in the past 15 years and have at least a 30 pack-year smoking history [2]. However, people who are not at higher risk of lung cancer, lung cancer must be screened using chest X ray (CXR) in residential or workplace health examinations. When previous CXR had old inflammatory shadows such as tuberculosis, calcification, and fibrotic change, it would become increasingly difficult to detect newly occurring lung cancer.

A comparative review of the CXR with the previous one helps in the detection of a new shadow. Pts who undergo repeat health checkups at the same facility can receive a comparative review of the CXR. It has been hypothesized that the diagnosis of CXR abnormalities in screening would be different when compared to previous CXR and when not. To clarify this, we conducted a retrospective cohort study in a large Japanese population to clarify the merits of comparative review in CXR, as well as a variety of chest abnormalities in CXR screening.

${ }^{\star}$ Correspondence to: Tatsuo Kimura, M.D. Ph.D., Department of Premier Preventive Medicine, Graduate School of Medicine, Osaka City University, 1-143 AbenoHARUKAS 21F, Abenosuji, Abeno-ku Osaka 545-6090, Japan, Tel: 81-6-6624-4041; Fax: 81-6-6624-8212; E-mail: kimutats@med.osaka-cu.ac.jp

Key words: health check-up,, lung cancer screening, CXR abnormalities, repeater Received: April 17, 2021; Accepted: April 26, 2021; Published: April 29, 2021 


\section{Participants and methods}

\section{Study population}

We analyzed one-year cohort derived from the ongoing MedCity 21 health examination registry from January 1 to December 31, 2019. Participants aged 20 years or older who were receiving a course including CXR were enrolled. Our clinic, "MedCity21," is a university outpatient clinic which performs complete health check-up. Pts with abnormalities that require medical care detected on CXR were immediately announced by call for recommendation for further examination and encouraged to visit our facility to undergo further examination by chest CT scan. The pts who previously visited MedCity21 to undergo a health check-up with CXR were called "repeated pts," and pts who visited MedCity 21 for the first time were called "first-visit pts." This study was conducted in accordance with the tenets of the Declaration of Helsinki. The registry protocol of the MedCity21 health examination and this retrospective observational study protocol was approved by the Ethics Committee of Osaka City University Graduate School of Medicine (approval No. 2927 and No. 2020-087, respectively). We performed an opt-out option, as explained in the instructions posted on the website of our facility.

\section{Clinical assessment}

CXR was performed using a direct radiography system (Radnext 50; Hitachi Healthcare Systems Inc., Tokyo, Japan) in the posterior-anterior $(120 \mathrm{kV}, 200 \mathrm{~mA})$ and lateral positions $(120 \mathrm{kV}, 320 \mathrm{~mA})$. Two doctors, board-certified in internal medicine specialty, respiratory medicine, and oncology, reviewed the CXR results using $3 \mathrm{M}$ monochrome monitor (RadiForce GX340, EIZO Corporation, Ishikawa, Japan). For baseline screening, the results of the initial CXR were categorized into five categories according to the criteria of the Japan Society of Ningen Dock [3]: A: Normal. B: Slightly abnormal. Nothing to worry about at present, or merely a deviation from normal that is not detrimental to your health. C: Require follow-up. There is a slight problem, but it is not serious now, and if you make the necessary changes, it is easily treated. D: Medical care needed. and E: On treatment. The abnormal shadows of category D in lung fields were classified into eight items, and those outside the lung field were classified into two categories. The items included nodular shadow $5 \mathrm{~mm}$ in diameter, ground glass opacity (GGO), granular shadow, reticular or interstitial shadow, cystic shadow, linear shadow including atelectasis, infiltration shadow, and pleural sign (pleural thickening, effusion, and pneumothorax), mediastinum abnormality, and cardiovascular system.

CT images were reviewed by two doctors certified as radiologists or oncologists. A CT device (Supria, Hitachi Healthcare Systems Inc., Tokyo, Japan) was used at $120 \mathrm{kV}, 30-250 \mathrm{~mA}$ of normal dose, scan time $0.75 \mathrm{~S} / \mathrm{rot}, 1.25$ times 16 collimation, table pitch of 1.3125 , slice/interval $5 / 5 \mathrm{~mm}$, and the same monitor as described above. The abnormalities in CXR were diagnosed by CT and classified into 10 items and others: old inflammatory change, nodules including GGO, granular shadow, acute inflammatory change, interstitial lung disease, pleural disease, nipples, bone shadow including bone island in the rib and spine of vertebrae, cardiovascular system, and soft tissue (fat, muscle, etc.). If an abnormality was noted on CXR but not on CT, it was classified as "no abnormality".

Patients with small nodules should undergo tailored follow-up according to guidelines [4]. Briefly, in the CT scans, if all solid or partly solid noncalcified pulmonary nodules or GGOs were less than $10.0 \mathrm{~mm}$ in diameter, a follow-up CT examination was performed 3 months later. On CT, none of the nodules grew over $10.0 \mathrm{~mm}$ in diameter, and CT was repeated 6 months later. In principle, a patient with a nodule 10 $\mathrm{mm}$ in diameter or larger, or a pleural sign, was referred to a specialized outpatient at Osaka City University Hospital or another equivalent hospital for further examinations. In the case of other diseases, we referred pts to the appropriate hospitals. In each case, doctors conferred with patients to decide on a course of action. Follow-up data were retrieved from the MedCity 21 health examination registry and referral letters from other hospitals at approximately 1 year of complete follow-up.

\section{Statistical analysis}

The baseline characteristics of the repeated and first-visit patients were compared using a chi-squared test. We also compared the varieties of shadows on CT scans between repeat and first-visit pts using chisquare tests. Statistical significance was set at $\mathrm{p}<0.05$. Statistical analyses were conducted using GraphPad PRISM for Windows version 5.0.1 software (GraphPad Software, San Diego, CA, USA).

\section{Results \\ Participant characteristics}

A total of 13,690 pts was enrolled, including 6,622 men (48.4\%) and 7,068 women (51.6\%) (Table 1). Pts ages ranged from 20 to 90 years, with a median age of 51 years, and the 25-75 percentile was 43-60 years. Four lung cancer cases were found. One was male and three were female. One of them was a smoker. The detection rate was 29.2 / 100,000. All cases were resected and revealed stage I adenocarcinoma. There were 7816 (57.1\%) non-smokers, 3821 (27.9\%) ex-smokers, 1821 (13.3\%) smoked pts less than 20 cigarettes per day, and 232 (1.7\%) smoked pts more than 21 cigarettes per day (15.0\%). Smoking was significantly less common among women than among men $(\mathrm{p}<0.01)$.

The numbers of repeated and first-visit pts were 7,872 (57.5\%) and $5,818(42.5 \%)$, respectively (Figure 1). Category D in CXR included a total of $334(2.4 \%)$ of 143 repeated pts and 191 first-visit pts. Repeated pts had a low proportion of abnormalities requiring further examination $(p<0.01)$. After the announcement by call for recommendation for further examination, a total of 234 pts $(70.1 \%)$ of $108 / 126$ patients received chest $\mathrm{CT}$ scans in our facility.

\section{Abnormalities detected by chest $\mathrm{X}$ ray}

Figure 2a shows the classification of CXR abnormalities by repeated and first-visit pts. A total of 234 of 108 repeated and 126 firstvisit pts who underwent chest CT scans in our clinic were analyzed for abnormalities. Nodular shadows were the most frequently noted shadows on CXR in both repeated (70.4\%) pts and first-visit (71.4\%) pts. The second most frequently noted shadow was a GGO in both repeated $(12.0 \%)$ and first-visit $(8.7 \%)$ pts. The following were granular

Table 1. Participant's characteristics

\begin{tabular}{|c|c|c|c|c|c|c|}
\hline \multicolumn{3}{|c|}{ 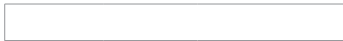 } & All & Male & Female & $\mathbf{p}$ \\
\hline \multicolumn{3}{|l|}{$\mathrm{N}$} & 13690 & 6622 & 7068 & \\
\hline \multirow{4}{*}{ Age } & \multicolumn{2}{|c|}{ median (range) } & $51(20-90)$ & 52 & 50 & N.S. \\
\hline & \multicolumn{2}{|c|}{$25-75$ percentile } & $43-60$ & & & \\
\hline & $\leq 65$ & (lung cancer) & 11918 & $5621(1)$ & 6297 & \\
\hline & $>65$ & (lung cancer) & 1772 & 1001 & $771(3)$ & \\
\hline \multicolumn{7}{|c|}{ Tabacco use } \\
\hline \multicolumn{2}{|c|}{ Never smoked } & (lung cancer) & 7816 & 2341(1) & $5475(2)$ & $<0.01 *$ \\
\hline \multicolumn{2}{|c|}{ Past smoker } & & 3821 & 2728 & 1093 & \\
\hline \multicolumn{2}{|c|}{$\begin{array}{l}\text { Current smoker } \\
1-20 / \text { day }\end{array}$} & (lung cancer) & 1821 & 1337 & $484(1)$ & \\
\hline \multicolumn{2}{|c|}{$\begin{array}{l}\text { Current smoker } \\
21</ \text { day }\end{array}$} & & 232 & 216 & 16 & \\
\hline
\end{tabular}




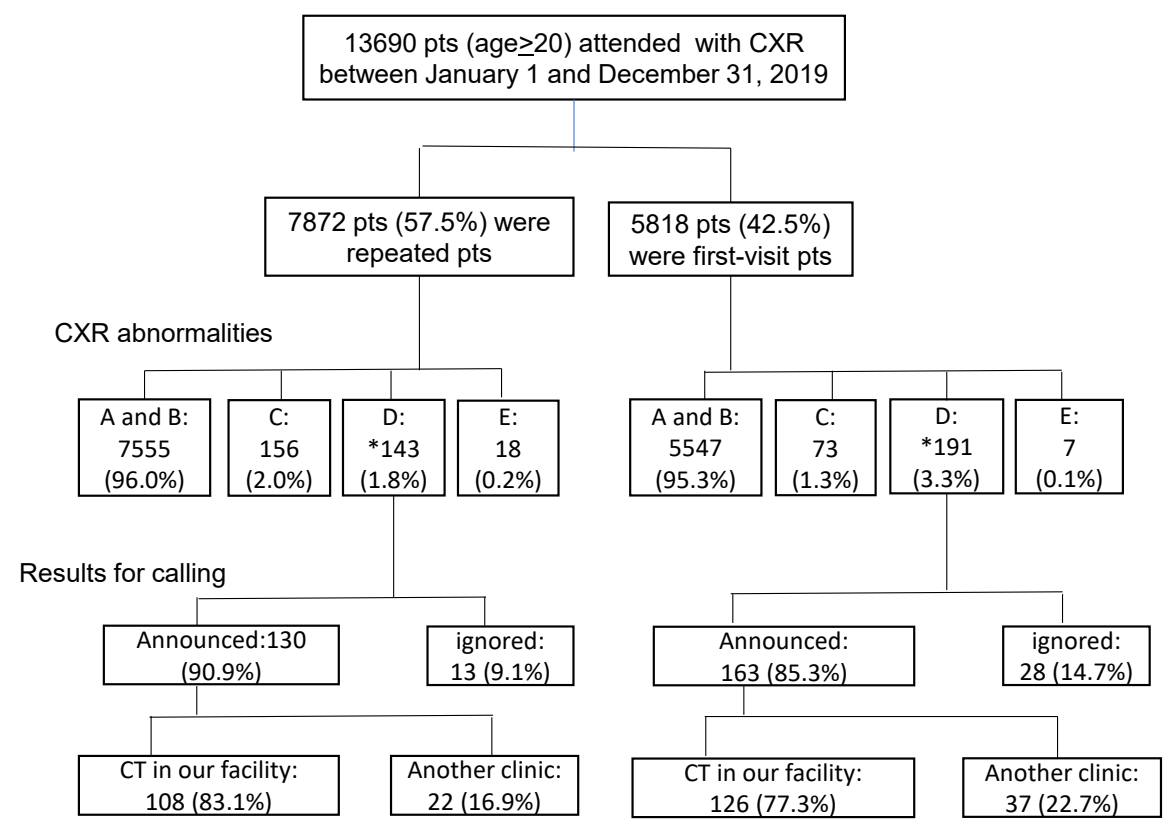

Figure 1. The number of repeated and first-visit participants with chest $\mathrm{X}$ ray abnormalities requiring further examination A: Normal. B: Slightly abnormal. C: Require follow-up. D: Medical care needed; E: On treatment

*Repeated pts had a low proportion of abnormalities requiring further examination $(\mathrm{p}<0.01)$.

a

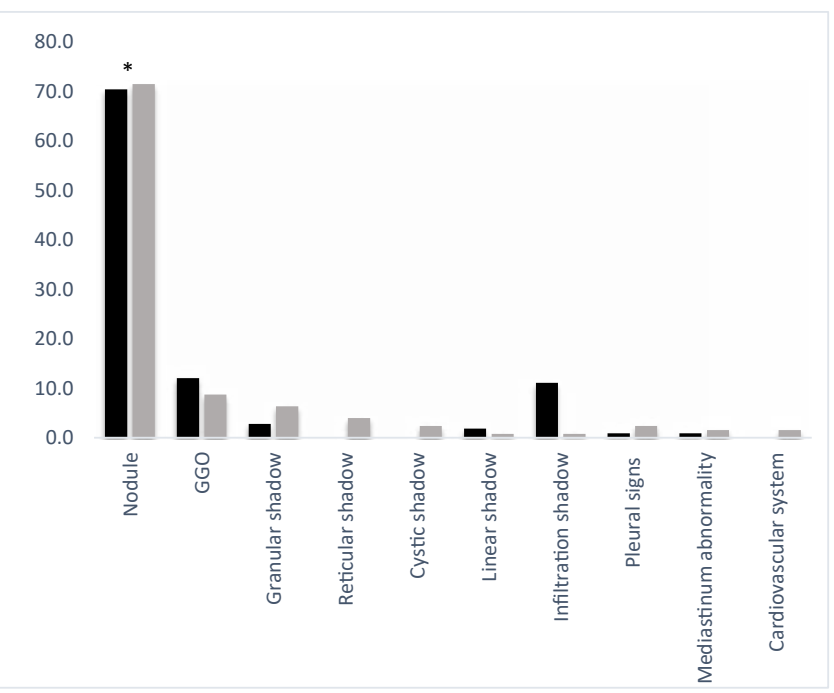

b

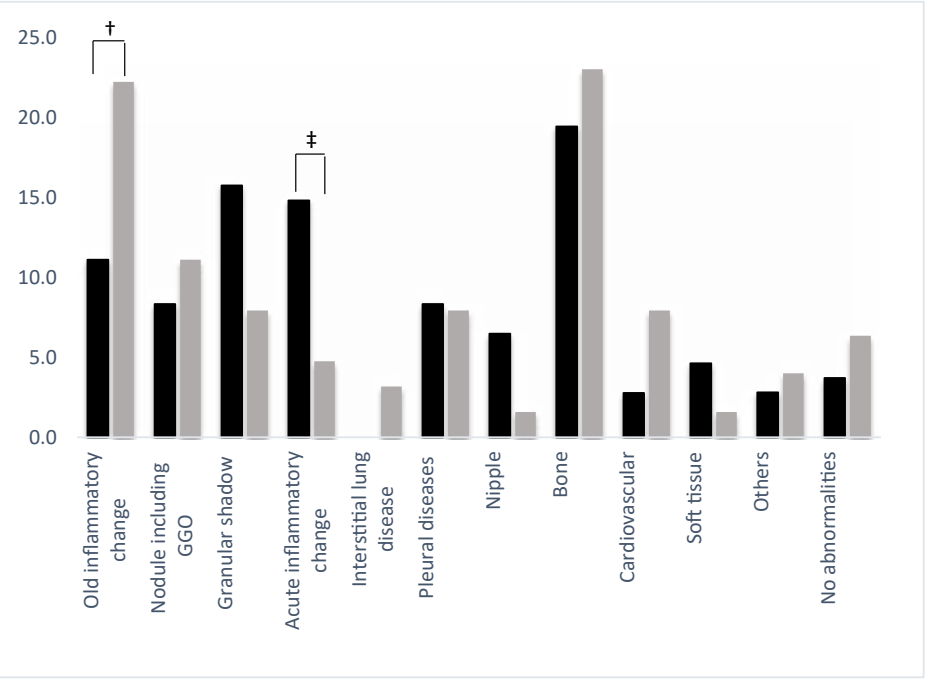

Repeated pts

First-visit pts

Figure 2. Classification of CXR abnormalities that required further examination and diagnoses by chest CT

The black and gray bars represent repeated and first-visit participants (pts), respectively. The abnormalities detected by CXR were classified into ten items (a) The most frequently noted shadows were nodular shadows in repeated $(70.4 \%)$ and first-visit $(71.4 \%)$ pts $(* p<0.01)$. CT scan revealed that the CXR abnormalities could be classified into 12 items. (b) Repeated pts had a significantly lower rate of old inflammatory change of $11.1 / 22.2 \%\left({ }^{\ddagger} \mathrm{p}=0.02\right)$ and a higher rate of acute inflammatory shadow of $14.8 / 4.8 \%(\ddagger \mathrm{p}=0.02)$ than first-visit pts.

$2.8 / 6.3 \%$, reticular $0.0 / 4.0 \%$, cystic $0.0 / 2.4 \%$, linear $1.9 / 0.8 \%$, infiltration $11.1 / 0.8 \%$, pleural $0.9 / 2.4 \%$, mediastinum $0.9 / 1.6 \%$, and cardiovascular $0.0 / 1.6 \%$, respectively. There was no significant difference in the distributions between the repeated and first-visit pts.

\section{Diagnoses by chest CT}

CT revealed CXR abnormalities (Figure $2 b$ ). CT scan revealed that the most frequently noted shadow was a bone shadow in both sets of pts. The second most frequently noted shadow was a granular shadow in repeated pts and old inflammatory changes in first-visit pts. The third most frequently noted shadow was acute inflammatory change in repeated pts and nodules, including GGO in first-visit pts. Repeated pts had a significantly lower proportion of old inflammatory changes $(\mathrm{p}=0.02)$, and a higher proportion of acute inflammatory shadow than first-visit pts $(\mathrm{p}=0.02)$. The percentages of diagnosis of repeated/ first-visit pts on chest CT were as follows: old inflammatory 11.2 / 
$22.2 \%$, nodules including GGO 8.3 / 11.1\%, granular $15.7 / 7.9 \%$, acute inflammatory 14.8 / 4.8\%, interstitial lung disease $0.0 / 3.2 \%$, pleural 8.3 / 7.9\%, nipple 6.5 / 1.6\%, bone 19.4 / 23.0\%, cardiovascular 2.8 / 7.9\%, soft tissue 4.6 / 1.6\%, others 2.8 / 4.0\%, and no abnormality 3.7 / $6.3 \%$, respectively.

\section{Discussion}

Repeated pts had a significantly lower proportion of abnormalities requiring further examination in CXR than the first-visit pts. Immediate confirmation by CT revealed that CXR abnormalities in repeated pts were diagnosed with different variations compared to those of firstvisit pts. On CXR as category D, the repeated pts had a significantly lower proportion of old inflammatory changes and a higher proportion of acute inflammatory shadows than first-visit pts. This means that the repeated pts may benefit from a decrease in false-positives and an increase in true-positives.

For a comparative review of the previous CXR, annual visits to health check-ups are recommended. A visit interval may be estimated by the speed of tumor growth based on tumor doubling time. In a review of the natural history of lung cancer over time, the mean tumor volume doubling time is approximately 135 days for patients diagnosed during routine medical care, 150 days in screening studies involving CXR, and 480 days in screening studies involving CT [5]. Most reports show doubling times in the range of 100 to 300 days for lung cancer [6,7]. In fact, in our study, all lung cancer cases were resected and revealed stage I adenocarcinoma. Subsolid pulmonary nodules, comprising pure GGO and part-solid nodules, have a high risk of indolent malignancy [8]. Indeed, in the extended follow-up of the Mayo Lung Project (MLP), the median survival for patients with resected early-stage disease was 16.0 years in the intervention arm, versus 5.0 years in the usual-care arm, with better survival for individuals in the intervention arm [9].

In our study, CT revealed that the most frequent abnormality was bone shadow. The second most frequent abnormality was granular shadows in repeated pts and old inflammatory changes in first-visit pts. Bone shadows may or may not be visible, depending on the slight difference in body position for CXR. A granular shadow due to old tuberculosis may not change over many years. Indeed, the prostate, lung, colorectal, and ovarian cancer screening trial revealed the most common non-cancer abnormalities in CXR were granuloma (10.7\%), scarring/ pulmonary fibrosis (8.2\%), and bone/soft tissue lesions (5.5\%) [10].

In our study, the lung cancer detection rate was $29.2 / 100,000$ and this was small compared to the incidence rate of $99.1 / 100,000$ in 2017 [11]. There may be several reasons for this. First, after the announcement by call for recommendation for further examination, about $70 \%$ of the patients with CXR abnormalities in both groups visited our facility for CT scan. The remaining $30 \%$ ignored the announcement or were observed by another clinic; therefore, we could not determine a diagnosis. Second, the repeated pts exceeded the majority and had a significantly lower rate of CXR abnormalities than the first-visit patients. The lung cancer incidence among repeated pts may be lower than the statistical data. Third, since the incidence of lung cancer is extremely low, the number of lung cancer cases is small and within the margin of error.

Several limitations must be considered when interpreting our study results. This was a one-year, single-center study. However, in our facility, the 2018 cohort had no significant differences compared to the 2019 cohort in terms of participants characteristics and the distribution of shadows in CXR and diagnoses by CT, which were reported previously
[12]. Second, a referral bias was considered. The repeated pts included those who had been seen three or more times. Third, CT was not included in the primary screening content in this study. Public lung cancer screening has aimed at reducing mortality in the population, but a self-paying optional complete health check-up aims for longevity in the individual. In future, in Japan, since health insurance provides additional support, most CT scans will be added. Fourth, screening tests can yield false-negative results. If we miss it once, subsequent misidentification is more likely.

In conclusion, immediate confirmation by CT revealed a difference in the proportion of CXR abnormalities between repeated and first-visit patients. We believe that regular physical examinations performed at the same facility are highly effective in ruling out lung cancer. A comparative review of previous CXR may be highly effective in avoiding unnecessary further examination. This will help improve lung cancer screening.

\section{Author Contributions}

\section{Conceptualization: Tatsuo Kimura,}

Data curation: Tatsuo Kimura, Shinya Fukumoto, Akemi Nakano, Hideki Fujii, Yuji Nadatani, Yukie Tauchi, Shingo Takashima, Yuko Nishii, Koji Otani, Naomi Ageshio

Supervision: Shinya Fukumoto, Yuji Nadatani, Norifumi Kawada,

Formal analysis: Naomi Ageshio, Tomohiro Suzumura,

Methodology: Tatsuo Kimura

Writing original draft: Tatsuo Kimura

Writing-review and editing: Tatsuo Kimura, Shinya Fukumoto, Akemi Nakano, Hideki Fujii, Yuji Nadatani, Yukie Tauchi, Shingo Takashima, Yuko Nishii, Koji Otani, Tomohiro Suzumura

Project administration: Tatsuo Kimura

\section{Acknowledgements}

The authors thank Mrs. Utsunomiya, Mr. Kusumi, and Mr. Miyao for help in data collection and Mrs. Sakama for secretary work.

\section{Financial support statement}

This work was supported by a Grant-in-Aid for Scientific Research (C) from JSPS KAKENHI 19K10579.

\section{Conflict of interest statement}

The authors declare that they have no financial conflicts of interest.

\section{References}

1. https://www.cancer.gov/types/lung/patient/lung-prevention-pdq

2. Wender R, Fontham ET, Barrera E, Colditz GA, Church TR, et al. (2013) American Cancer Society lung cancer screening guidelines. CA Cancer J Clin 63: 107-117. [Crossref]

3. https://www.ningen-dock.jp/en/other/inspection

4. Wood DE, Kazerooni EA, Baum SL, Eapen GA, Ettinger DS, et al. (2018) Lung Cancer Screening, Version 3.2018, NCCN Clinical Practice Guidelines in Oncology. $J$ Natl Compr Canc Netw 16: 412-441. [Crossref]

5. Detterbeck FC, Gibson CJ (2008) Turning gray: the natural history of lung cancer over time. J Thorac Oncol 3: 781-792. [Crossref]

6. Usuda K, Saito Y, Sagawa M, Sato M, Kanma K, et al. (1994) Tumor doubling time and prognostic assessment of patients with primary lung cancer. Cancer 74: 22392244. [Crossref] 
7. Yankelevitz DF, Kostis WJ, Henschke CI, Heelan RT, Libby DM, et al. (2003) Overdiagnosis in chest radiographic screening for lung carcinoma: frequency. Cancer 97: 1271-1275. [Crossref]

8. Hammer MM, Palazzo LL, Kong CY, Hunsaker AR (2019) Cancer Risk in Subsolid Nodules in the National Lung Screening Trial. Radiology 293: 441-448. [Crossref]

9. Marcus PM, Bergstralh EJ, Fagerstrom RM, Williams DE, Fontana R, et al. (2000) Lung cancer mortality in the Mayo Lung Project: Impact of extended follow-up. $J$ Natl Cancer I 92: 1308-1316. [Crossref]
10. Pinsky PF, Freedman M, Kvale P, Oken M, Caporaso N, et al. (2006) Abnormalities on chest radiograph reported in subjects in a cancer screening trial. Chest 130: 688-693. [Crossref]

11. https://ganjoho.jp/reg_stat/statistics/dl/index.html\#mortality

12. Kimura T, Fukumoto S, Nakano A, Fujii H, Nadatani Y, et al. (2020) Repeat lung cancer screenings by chest $\mathrm{X}$ ray have a profit for the avoidance of further examinations (abstract number: OOP-5-1). In: The 27th International Health Evaluation and Promotion Association and The 4th World Congress on Ningen Dock, Yokohama, Japan.

Copyright: $@$ 2021 Kimura T. This is an open-access article distributed under the terms of the Creative Commons Attribution License, which permits unrestricted use, distribution, and reproduction in any medium, provided the original author and source are credited. 